J. Clin. Chem. Clin. Biochem.

Vol. 14, 1976, pp. 15-22

\title{
Beitrag zur Charakterisierung des Oxidoreductase-Inhibitors
}

\author{
Von H. Gallati
}

Diagnostische Forschungsabteilung der F. Hoffmann-La Roche \& Co. AG, Basel

(Eingegangen am 21. August/23. Oktober 1975)

Zusammenfassung: Der Oxidoreductase-Inhibitor wird aus NAD im alkalischen Milieu hergestellt und chromatographisch gereinigt. Neben den Extinktions- und Fluoreszenzspektren werden auch die Stabilität des Inhibitors sowie seine Aktivitätshemmung in Abhängigkeit der Enzym- und Coenzymkonzentration untersucht. Auf Grund der kinetischen Studien kann gesagt werden, daß es sich beim Oxidoreductase-Inhibitor um einen nicht-kompetitiven Hemmstoff handelt.

\section{Characterization of the oxidoreductase inhibitor}

Summary: The oxidoreductase inhibitor was prepared from NAD in alkaline solution, and purified chromatographically. Investigations are reported on the extinction and fluorescence spectra, stability of the inhibitor, and the dependence of inhibition on the concentration of enzyme and coenzyme. Kinetic studies show that the inhibition is non-competitive.

\section{Einfuihing}

Bei der Aktivitätsbestimmung einiger Oxidoreductasen muß beachtet werden, daß im eingesetzten NADH ein Inhibitor enthalten sein kann, der schon in kleinsten Konzentrationen die betreffende Enzymaktivität wesentlich zu hemmen vermag.

Verschiedentlich wurde dieser Oxidoreductase-Inhibitor aus NADH-Präparaten mit Hilfe einer DEAE-CelluloseChromatographiè angereichert (1-7), um anschließend diese Substanz zu charakterisieren. Dabei wurde festgestellt, daß

1. das Extinktionsspektrum des Oxidoreductase-Inhibitors demjenigen des NADH șehr ähnlich ist. Es ergeben sich auch beim Inhibitor die beiden Extinktionsmaxima bei den Wellenlängen $340 \mathrm{~nm}$ und $260 \mathrm{~nm}(1,2$, $4,6,8)$.

2. der Oxidoreductase-Inhibitor bei einer Anregung von $366 \mathrm{~nm}$ in gleicher Weise fluoresżiert wie das NADWH $(2,7)$.

3. für den Inhibitor ein Molekulargewicht von 700 angenommen werden kann (4).

4. der Oxidoreductase-Inhibitor gegenüber Säure so instabil ist wie das $\mathrm{NADH}(1,2)$.

5. es sich beim Oxidoreductase-Inhibitor um einen kompetitiven Hemmstoff handelt $(1,3,4)$. Diese Substanz zeigt allerdings eine bedeutend höhere Substrataffinität als das NADH. Gegenüber der Lactat-Dehydrogenase aus Kaninchenmuskeln errechnete Strandjord (4) eine Inhibitorkonstante $\left(\mathrm{K}_{\mathbf{i}}\right)$ von $22 \mu \mathrm{mol} / 1$ für den Oxidoreductase-Hemmstoff.

6. dieser Inhibitor die einzelnen Oxidoreductasen in sehr unterschiedlichem Maß hemmt $(1,5,9)$.

In einer ersten Arbeit (10) wurde aufgezeigt, daß der Oxidore ductase-Inhibitor nicht aus dem NADH, sondern nur aus dem NAD im alkalischen Milieu gebildet wird, und es wurden günstige Bedingungen zur Bildung und eine einfache Methode zur Reinigung dieses Inhibitors beschrieben.

Die vorliegende Arbeit möchte ein Beitrag zur Charakterisierung des gereinigten Oxidoreductase-Inhibitors sein. Dabei wurde vor allem die Frage abgeklärt, ob es sich bei diesem Imhibitor um einen kompetitiven oder um einen nichtkompetitiven Hemmstoff handelt.

\section{Material und Methoden}

1. Die Enzyme Creatin-Kinase (EC 2.7.3.2; Kaninchenmuskel), Glucose-6-phosphat-Dehydrogenase (EC 1.1.1.49; Hefe), Glutamat-Dehydrogenase (EC 1.4.1.2; Rinderleber), MalatDehydrogenase, (EC 1.1.1.37) Glutamat-Oxalacetat-Transaminase (EC 2.6.1.1), Glutamat-Pyruvat-Transaminase (EC 2.6.1.2), Isoenzym 1 und 2 der Lactat-Dehydrogenase (EC 1.1.1.27; Schweineherz), Isoenzym 3, 4 und 5 der Lactat- 
Dehydrogenase (EC 1.1.1.27; Schweinemuskel) und SorbitDehydrogenase (EC 1.1.1.14; Schafsleber) sowic die Coenzyme NAD und NADH wurden von Boehringer Mannheim bezogen.

2. Für die chromatographische Reinigung des OxidoreductaseInhibitors wurde DEAE-Cellulose (Whatman DE-23) und Sephadex G-15 (Pharmacia) eingesetzt.

Die mit $10 \mathrm{mmol} / 1$ Natriumphosphat-Puffer, $\mathrm{pH} 8,5$ äquilibrierte DEAE-Cellulose wurde in eine Säule $(2,5 \times 40 \mathrm{~cm})$ gepackt und das Substanzgemisch mit dem Inhibitor aufgetragen. Die Fraktionierung erfolgte nach gutem Auswaschen mit einem linearen $\mathrm{NaCl}-\mathrm{Gradienten}$.

Sephadex G-15 wurde in $2 \mathrm{mmol} / \mathrm{l}$ Natronlauge aufgenommen und anschließend in eine Säule von $1 \times 450 \mathrm{~cm}$ gepackt.

Alle chromatographischen Arbeiten wurden bei Raumtemperatur durchgeführt.

3. Zur Bildung des Oxidoreductase-Inhibitors wurde $1 \mathrm{~g}$ NAD mit $2 \mathrm{~g}$ tri-Kaliumphosphat in einer Glasampulle verrieben und während 3 Tagen bei Raumtemperatur in wassergesättigter Atmosphäre inkubiert.

4. Zur Reinigung des Oxidoreductase-Inhibitors wurde das in tri-Kaliumphosphat inkubierte NAD in wenig dest. Wasser gelöst und auf DEAE-Cellulose aufgetragen. Die Fraktionen mit dem Inhibitor wurden gepoolt, eingeengt und anschliessend auf die Sephadex G-15-Säule aufgetragen. Die Fraktionen mit Inhibitor-Aktivität wurden vereinigt und nochmals unter gleichen Bedingungen auf DEAE-Cellulose und Sephadex G-15 chromatographiert.

Nach dieser chromatographischen Reinigung konnte die Aktivität der Lactat-Dehydrogenase zu 50\% gehemmt werden, wenn ihrer Testlösung $30 \mathrm{nmol} / 1$ Oxidoreductase-Inhibitor zugesetzt wurde. Der Konzentrationsangabe des Inhibitors liegt dabei die Annahme zugrunde, daß der molare Extinktionskoeffizient für das NAD und für den Inhibitor bei der Wellenlänge $260 \mathrm{~nm}$ identisch ist.

5. Die Extinktionsspektren wurden mit einem Spektralphotometer Beckman DB-G bei $25^{\circ} \mathrm{C}$ in Quarzküvetten von $10 \mathrm{~mm}$ Schichtdicke aufgenommen.

6. Die Fluoreszenzspektren wurden in $10 \times 10 \mathrm{~mm}$ Quarzküvetten auf einem AMINCO-BOWMAN-Spectrofluorometer, der an einen AMINCO-Photomultiplier-Microphotometer und einem AMINCO-X-Y-Schreiber angeschlossen ist, aufgenommen.

7. Die Testbedingungen zur Aktivitätsmessung der CreatinKinase, Glutamat-Dehydrogenase, Glutamat-Oxalacetat-Transaminase, Glutamat-Pyruvat-Transaminase und der LactatDehydrogenase entsprachen den Empfehlungen der Deutschen Gesellschaft für Klinische Chemie (11).

Zur Aktivitätsbestimmung der Glucose-6-Phosphat-Dehydrogenase, Malat-Dehydrogenase und der Sorbit-Dehydrogenase wurden Testkits von Boehringer verwendet.

\section{Resultate}

Extinktionsspektrum des Oxidoreductase-Inhibitors

Für das Absorptionsspektrum der Abbildung 1 wurde der gereinigte Oxidoreductase-Inhibitor in verdünnter Natronlauge (pH der Lösung 9,8) aufgenommen.

Das Spektrum zeigt ein Extinktionsmaximum bei der Wellenlänge $260 \mathrm{~nm}\left(\mathrm{E}_{260} \mathrm{~nm}=0.780\right)$ und ein Extinktionsminimum bei der Wellenlänge $230 \mathrm{~nm}\left(\mathrm{E}_{230 \mathrm{~nm}}=\right.$ 0,225). Im Bereich der Wellenlänge $390 \mathrm{~nm}$ bis $290 \mathrm{~nm}$ steigt die Extinktion nur unbedeutend an und erreicht bei der Wellenlänge $340 \mathrm{~nm}$ den Wert von 0.030 .

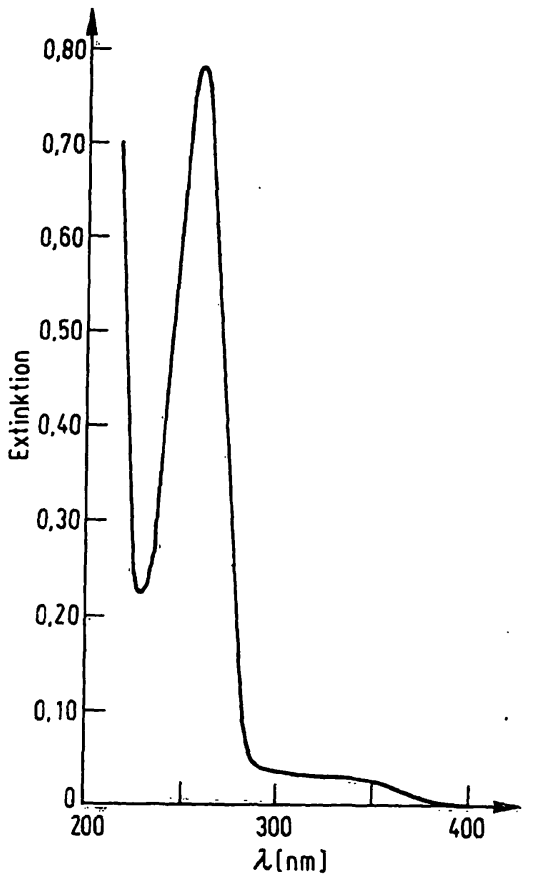

Abb. 1. Absorptionsspektrum des Oxidoreductase-Inhibitors. In einer Quarzküvette von $10 \mathrm{~mm}$ Schichtdicke wurde gereinigter Oxidoreductàse-Inhibitor zu $2 \mathrm{mmol} / 1 \mathrm{Na}$ tronlauge zugegeben und das Absorptionsspektrum dieser Lösung im Bereich der Wellenlänge $400 \mathrm{~nm}$ bis $220 \mathrm{~nm}$ aufgenommen.

Das , $260 \mathrm{~nm} / 340$ nm Extinktionsverhältnis“ des Oxido reductase-Inhibitors beträgt 26 . Für NADH beträgt das ,260 nm/340 nm Extinktionsverhältnis “ 2,27 (7).

\section{Fluoreszenzspektren des Oxidoreductase-Inhibi- tors}

In Abbildung 2 sind die Anregungs- und Emisșionsspektren des gereinigten Oxidoreductase-Inhibitors und zum Vergleich dazu die entsprechenden Spektren des NADH dargestellt. Dazu wurden beide Substanzen im alkalischen Milieu (Natronlauge, pH 10) gelöst. Unter diesen Bedingungen liegt das Anregungsmaximum für den Oxidoreductase-Inhibitor bei $340 \mathrm{~nm}$ und für das NADH bei $360 \mathrm{~nm}$. Die Emissionsspektren der beiden Substanzen erreichen das Maximum bei $460 \mathrm{~nm}$.

Die Abbildung 3 zeigt die Anregungs- und Emissionsspektren der beiden Substanzen, wenn sie in verdünnter Salzsäure $(\mathrm{pH}$ 2) gelöst werden. Während das NADH soğleich die Fluoreszenz verliert, ergibt sịch unter diesen Bedingungen für den Oxidoreductase-Inhibitor eine Fluoreszenz, die gegenüber jener im älkalischen Milieu wesentlich (etwia 40\%) stärker ist. Das Anregungsmaximum liegt bei $360 \mathrm{~nm}$ und das Maximum des Emissionsspektrums bei $470 \mathrm{~nm}$.

Es ist naheliegend, dieses unterschiedliche Verhalten der Fluoreszenz der beiden Substanzen im sauren Milieu für die Qualitätskontrolle des NADÄ nutzbar zu machen, indem in einer angesäuerten NADH-Lösung die Restfluoresżenz bestimmt und damit der Gehalt an Oxidore- 


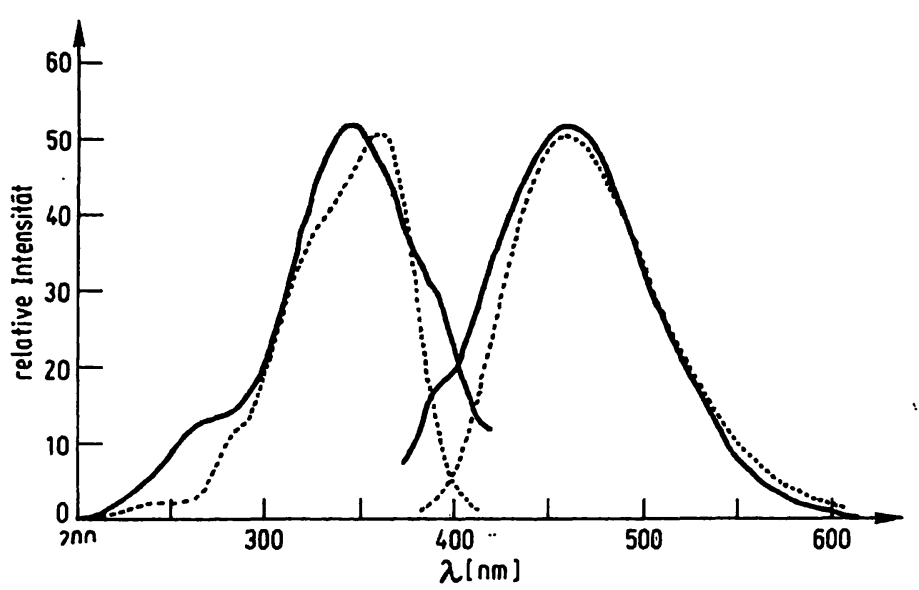

Abb. 2. Fluoreszenzspektren des Oxidoreductase-Inhibitors und des NADH im alkalischen Milieu.

In einem AMINCO-BOWMAN-Fluorometer wurden bei Raumtemperatur in einer Quarzkïvette von $10 \times 10 \mathrm{~mm}$ Schichtdicke die Anregungs- und Emissionsspektren der folgenden Lösungen aufgenommen:

a) $0,02 \mathrm{mmol} / 1$ gereinigter Oxidoreductase-Inhibitor in $2 \mathrm{mmol} / 1$ Natronlauge (meter multiplier: $0,01 / \mathrm{sen}$ sitivity: 15) (-)

b) $0,15 \mathrm{mmol} / 1 \mathrm{NADH}$ in $2 \mathrm{mmol} / 1$ Natronlauge (meter multiplier: $0,3 /$ sensitivity: 39$)(---)$

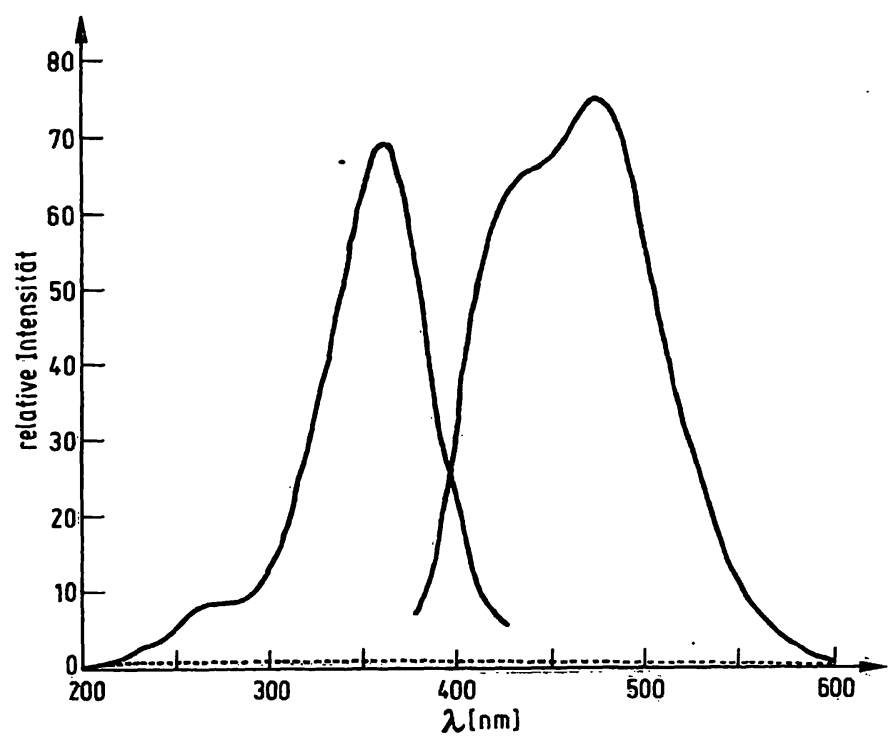

Abb. 3. Fluoreszenżspektrẹn des Oxidoreductase-Inhibitors uñ des NAD̂́H im sauren Milieu

Unter idèntischen Bedingungen wie für Abb. 2 wurden die Anregungs- und Emissionsspektreñ einer sauren Oxidoreductase-Iṇhibitorlösung $(0,02 \mathrm{mmol} / \mathrm{l})(-)$ sowie einer sauren NADH-Lösung $(0,15 \mathrm{mmol} / 1)(---)$ aufgenommen.

ductase-Inhibitor berechnet wird. Diesbezügliche Versuche haben aber ergeben, daß aus dem NAD, das zur Bildung des Inhibitors im alkalischen Milieu inkubiert wird, noch andere fluoreszierende Substanzen entstehen, die zwar keine Irikibitor-Aktivität aufweisen, deren Fluoreszenz aber auch im sauren Milieu erhalten bleibt.

\section{Stabilität des Oxidoreductase-Inhibitors}

Im sauren Milieu ist der Oxidoreductase-Inhibitor äußerst instabil; er verliert sogleich seine Hemmwirkung. Durch erneute Alkalisierung der angesäuerten Inhibitorlösung kann der Hemmstoff in seiner Wirksamkeit nicht reaktiviert werden, auch wenn diese alkalische Lösung längere Zeit bei verschiedenen Temperaturen inkubiert wird.

Im alkalischen Milieu zeigt der Oxidoreductase-Inhibitor eine erstaunlich gute Stabilität. In Abbildung 4 sind die Resultate zusammengestellt, wie sie bei einer Stabilitätsprüfung der alkalischen ( $50 \mathrm{mmol} / 1$ Phosphatpuffer, $\mathrm{pH}$ 8,5 ) Inhibitorlösung erhalten wurden. Daraus ist ersichtlich, daß während der Inkubationsdauer von 4 Wochen der Inhibitor bei den Inkubationstemperaturen zwischen $4^{\circ} \mathrm{C}$ und $60^{\circ} \mathrm{C}$ nur langsam seine Hemmwirkung verliert, während bei $70^{\circ} \mathrm{C}$ und $80^{\circ} \mathrm{C}$ der Inhibitor wesentlich schneller inaktiviert wird.

Der Oxidoreductase-Inhibitor ist in organischen Lösungsmitteln stabil. Zu diesem Versuch wurde der Inhibitor während 15 Minuten bei Raumtemperatur in den folgenden Lösungsmitteln vorinkubiert: Ethanol, Ethylenglykol, Aceton, Benzol, Oktanol, Toluol, n-Hexan, Diethylamin. Durch keines der angeführten organischen Lösungsmittel konnte der Inhibitor in seiner Hemmwirkung beeinträchtigt oder inaktiviert werden.

Einfluß verschiedener Zusätze auf die Hemmwirkung des Inhibitors

Der Einfluß verschiedener Zusätze auf die Hemmwirkung des Oxidoreductase-Inhibitors wurde untersucht, um ab-

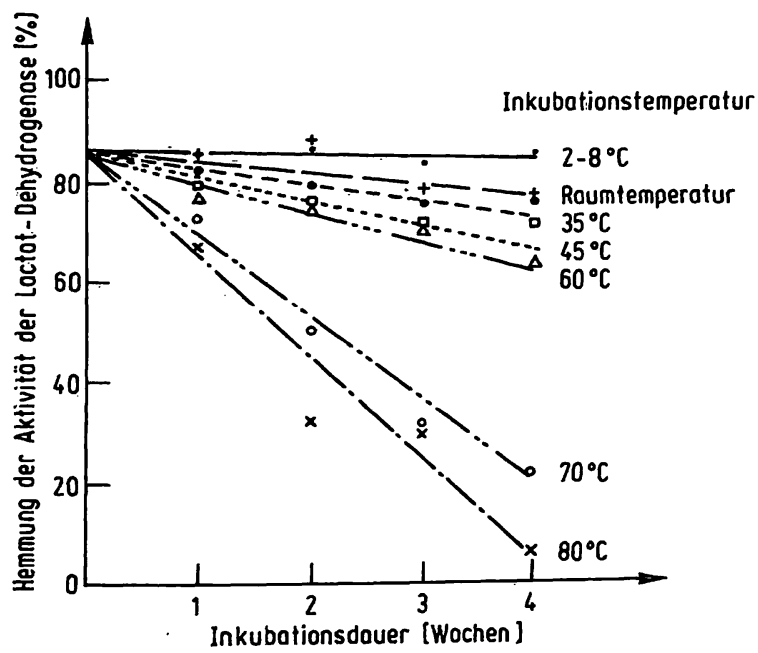

Abb. 4. Stabilität des Oxidoreductase-Inhibitors im alkalischen Milieu

Gereinigter Oxidoreductase-Inhibitor wurde in $50 \mathrm{mmol} / 1$ Natriumphosphatpuffer, pH 8,5 aufgenommen und die Lösung in 7 braune Glasampullen mit Schraubverschluß aufgeteilt. Je eine dieser Ampullen wurde bei den Temperaturen $2-8^{\circ} \mathrm{C}, 19-22^{\circ} \mathrm{C}, 35^{\circ} \mathrm{C}$, $45^{\circ} \mathrm{C}, 60^{\circ} \mathrm{C}, 70^{\circ} \mathrm{C}$ und $80^{\circ} \mathrm{C}$ inkubiert. Nach bestimmten Zeitintervallen wurde von jeder Ampulle je eine Probe entnommen und damit die Aktivitätshemmung der Lactat-Dehydrogenase bestimmt. 
zuklären, ob auf diese Weise die Aktivitätshemmung unterbunden werden kann.

Auf Grund der erhaltenen Resultate kann gesagt werden, daß Harnstoff (bis $740 \mathrm{mmol} / \mathrm{l}$ ), Thioharnstoff (bis $74 \mathrm{mmol} / \mathrm{l}$ ), Eisen-III-Ionen (bis $80 \mu \mathrm{mol} / \mathrm{l}$ ), Eisen-IIIonen (bis $10 \mathrm{mmol} / \mathrm{l}$ ), Magnesiumionen (bis $10 \mathrm{mmol} / \mathrm{l}$ ), Calciumionen (bis $10 \mathrm{mmol} / \mathrm{l}$ ), Zinkionen (bis $10 \mathrm{mmol} / \mathrm{l}$ ), Glycin (bis $50 \mathrm{mmol} / \mathrm{l}$ ), Histidin (bis $10 \mathrm{mmol} / \mathrm{l}$ ), Cystein (bis $10 \mathrm{mmol} / \mathrm{l})$, Cystein + Zinkionen $(1,5 \mathrm{mmol} / \mathrm{l})$, Histidin + Zinkionen $(1,5 \mathrm{mmol} / \mathrm{l})$ und Cystein + Histidin + Zinkionen $(1,5 \mathrm{mmol} / \mathrm{l})$ die Hemmwirkung des Oxidoreductase-Inhibitors in keiner Weise beeinflussen.

Aktivitätshemmung verschiedener Enzyme durch den Oxidoreductase-Inhibitor

Um den Grad der Aktivitätshemmung einzelner Enzyme durch den Oxidoreductase-Inhibitor zu messen, wurden zu den entsprechenden Testlösungen unterschiedliche Mengen des Inhibitors zugefügt und die einzelnen Resultate mit der Aktivität des inhibitorfreien Enzymtests (=100\%) verglichen. Die Resultate, die in der Tabelle 1 zusammengestellt sind, zeigen, daß der Inhibitor die Aktivität der einzelnen Oxidoreductasen sehr unterschiedlich hemmt. Die Glutamat-Dehydrogenase, Sorbit-Dehydrogenase und die Glucose-6-phosphat-Dehydrogenase werden auch durch eine höhere Inhibitorkonzentration in ihrer Aktivität nicht beeinträchtigt, während die Malat-Dehydrogenase nur schwach gehemmt wird. Hingegen werden alle 5 Isoenzyme der Lactat-Dehydrogenase durch den Oxidoreductase-Inhibitor stark gehemmt.

Wird für die Aktivitätsbestimmung eines Enzyms (z. B. Glutamat-Oxalacetat-Transaminase) die Test-Reaktion mit einer Oxidoreductase als Hilfs- oder Indikatorenzym gekoppelt, hat der Inhibitor solange keinen Einfluß auf den Test, als die Restaktivität der zum Teil gehemmten Oxidoreductase noch immer im Überschuß vorhanden ist.
Einfluß der Enzymkonzentration auf den Hemmungsgrad der Enzymaktivität bei konstanter Inhibitormenge

Der Grad der Aktivitätshemmung der Lactat-Dehydrogenase durch eine bestimmte Menge Oxidoreductase-Inhibitor ist abhängig von der eingesetzten Enzymkonzentration. Wie die Abbildung 5 zeigt, nimmt bis zu einem

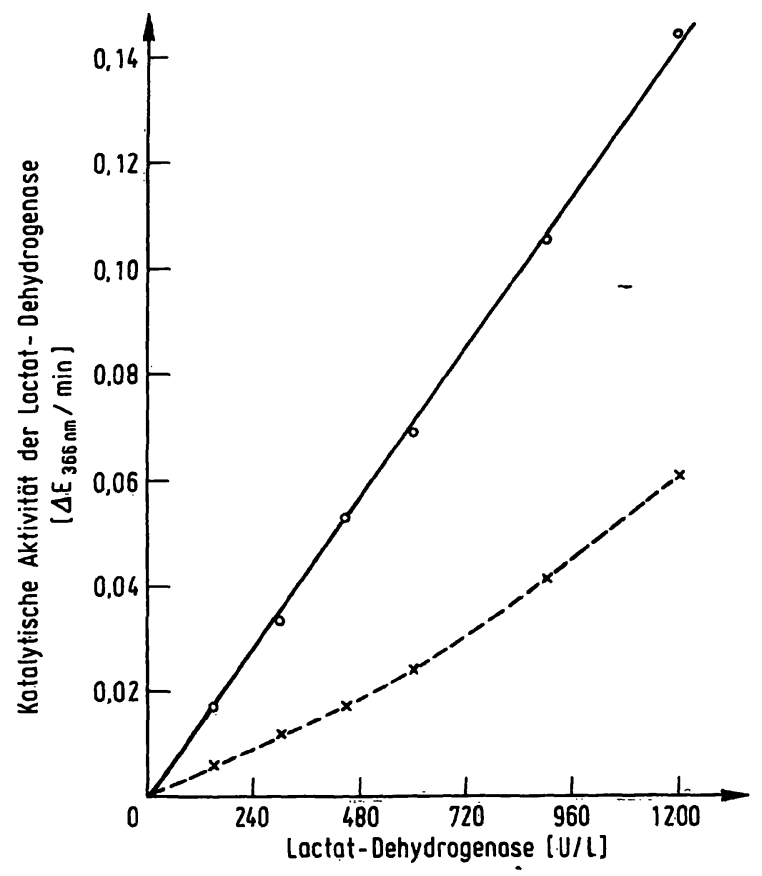

Abb. 5. Abhängigkeit der Aktivitätshemmung bei konstanter Inhibitormenge von der Lactat-Dehydrogenase-Konzentration

Eine Konzentrationsreihe von Enzymlösungen wurde in $50 \mathrm{mmol} / 1$ Natriumphosphatpuffer, $\mathrm{pH} 7,5$ hergestellt und die Aktivität der einzelnen Lösungen ohne Inhibitor (-) sowie bei Anwesenheit von $0,05 \mu \mathrm{mol} / 1$ Oxidoreductase-Inhibitor $(x---x)$ bei der Inkubationstemperatur von $25^{\circ} \mathrm{C}$ bestimmt, indem die Extinktionsdifferenz pro Minute bei der Wellenlänge $366 \mathrm{~nm}$ $\left(\Delta \mathrm{E}_{366 \mathrm{~nm} / \mathrm{min}}\right)$ gemessen wurde.

Tab. 1. Aktivitätshemmung verschiedener Enzyme durch den Oxidoreductase-Inhibitor

Zur Aktivitätsbestimmung der einzelnen Enzyme wurde zu den entsprechenden Testlösungen Oxidoreductase-Inhibitor in den angegebenen Konzentrationen zugemischt.

Hemmung der Enzym-Aktivität [\%] bei folgenden Konzentrationen des Oxidoreductase-Inhibitors

\begin{tabular}{|c|c|c|c|c|}
\hline Enzym & $0,025 \mu \mathrm{mol} / \mathrm{l}$ & $0,05 \mu \mathrm{mol} / 1$ & $0,125 \mu \mathrm{mol} / 1$ & $0,25 \mu \mathrm{mol} / \mathrm{l}$ \\
\hline Glutamat-Dehydrogenase & 0 & 0 & 0 & 0 \\
\hline Sorbit-Dehydrogenase & 0 & 0 & 0 & 0 \\
\hline Glucose-6-phosphat-Dehydrogenase & 0 & 0 & $\dot{\theta}$ & 0 \\
\hline Malat-Dehydrogenase & 0 & 4 & 12 & 19 \\
\hline Lactat-Dehydrogenase Isoenżym 1 & 32 & 56 & 76 & 85 \\
\hline Lactat-Dehydrogenase Isoenzym 2 & 25 & 51 & 81 & 90. \\
\hline Lactat-Dehydrogenase Isoenzym 3 & 28 & 50 & 72 & 85 \\
\hline Lactat-Dehydrogenase Isoenzym 4 & 28 & 44 & 75 & 86 \\
\hline Lactat-Dehydrogenase Isoenzym 5 & 27 & .40 & 69 & 84 \\
\hline Glutamat-Pyruvat-Transaminase & 0 & 0 & 0 & 0 \\
\hline Glutamat-Oxalacetat-Transaminase & 0 & 0 & 0 & 0 \\
\hline Creatin-Kinase & 0 & 0 & 0 & 0 \\
\hline
\end{tabular}


Lactat-Dehydrogenase-Gehalt von 1200 U/1 die Extinktionsdifferenz pro Minute $\left(\Delta \mathrm{E}_{366} \mathrm{~nm} / \mathrm{min}\right)$ linear $\mathrm{zu}$, wenn der Oxidoreductase-Inhibitor in der Testlösung fehlt. Wird die Lactat-Dehydrogenase-Testlösung mit $0,05 \mu \mathrm{mol} / 1$ Inhibitor versetzt, nimmt die Extinktionsdifferenz ( $\left.\Delta \mathrm{E}_{366 \mathrm{~nm}} / \mathrm{min}\right)$ mit höherem Lactat-Dehydrogenase-Gehalt stärker zu. Dies bedeutet, daß die proportionale Aktivitätshemmung der Lactat-Dehydrogenase mit steigender Enzymkonzentration abnimmt.

Zugabe von Rinderserum-Albumin in verschiedenen Konzentrationen zur Lactat-Dehydrogenase-Testlösung hat auf die Aktivitätshemmung keinen Einfluß.

\section{Einfluß des Oxidoreductase-Inhibitors auf die Hitzestabilität der Lactat-Dehydrogenase}

Bei einer Inkubationstemperatur von $65^{\circ} \mathrm{C}$ kann die Hitzestabilität der Lactat-Dehydrogenase sowohl durch Zusatz von NADH wie auch von Oxidoreductase-Inhibitor wesentlich verbessert werden, wie aus den Resultaten der Abbildung 6 ersichtlich ist. Nach einer Inkubationsdauer von $20 \mathrm{~min}$ bei einer Inkubationstemperatur von $65^{\circ} \mathrm{C}$ beträgt die Restaktivität der Lactat-Dehydrogenase ohne Zusatz

mit $250 \mu \mathrm{mol} / 1 \mathrm{NADH}$

mit $50 \mathrm{nmol} / 1$ Inhibitor
$22 \%$

$75 \%$ der anfänglich vorhandenen Lactat-Dehydrogenase-Aktivität. Mit einer gegenüber dem NADH 5000-fach kleineren Konzentration an Oxidoreductase-Inhibitor läßt sich also eine vergleichbare Hitzestabilisierung der LactatDehydrogenase erreichen.

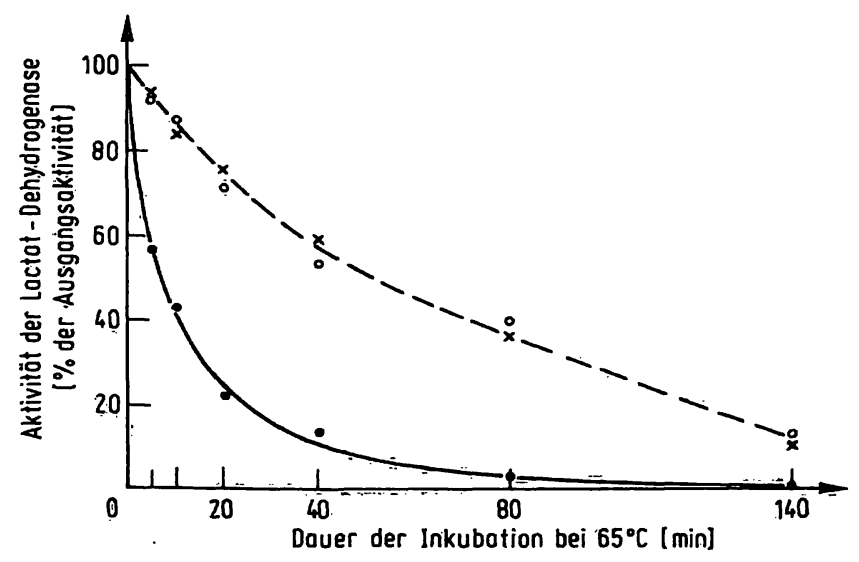

Abb. 6. Einfluß des Oxidoreductase-Inhibitors auf die Hitzestabilität der Lactat-Dehydrogenașe

Enzymlösung in 0,1 mol/1 Natriumphosphatpuffer, pH 7,5 wurde ohne Zusatz (.- $\cdot$ ) mit $250 \mu \mathrm{mol} / 1$ NADH (0- - 0$)$ und mit $50 \mathrm{nmol} / 1$ OxidoreductaseInhibitor $(x---x)$ im Wasserbad von $65^{\circ} \mathrm{C}$ inkubiert. Nach bestimmten Zeitintervallen wurde von den einzel: nen Enzymlösungen eine Probe entnommen und damit die Aktivität der Lactat-Dehydrogenase bestimmt.
Einfluß von NADH auf die Aktivitätshemmung des Oxidoreductase-Inhibitors

Wird der Oxidoreductase-Inhibitor zur Lactat-Dehydrogenase-Testlösung zugemischt und die Reaktion durch Zugabe des Enzyms gestartet, so ist eine Aktivitätshemmung festzustellen, die anfänglich noch klein ist. Sie nimmt dann rasch zu und erreicht nach etwa 5 Minuten ihre volle Wirksamkeit.

Wird aber die Lactat-Dehydrogenase mit der entsprechenden Menge Inhibitor einige Minuten vorinkubiert, und die Enzymreaktion durch die Zugabe von NADH gestartet, so ist von Anfang an die volle Aktivitätshemmung vorhanden. Wie die Abbildung 7 zeigt, genügt eine 10minütige Vorinkubation der Lactat-Dehydrogenase mit dem Inhibitor, um die volle Aktivitätshemmung des Enzyms vom Reaktionsbeginn an zu garantieren. Eine noch längere Vorinkubationsdauer erhöht die Hemmwirkung der Anfangs-Aktivität nur unwesentlich.

EinfluB der NADH-Konzentration auf den Grad der Aktivitätshemmung der Lactat-Dehydrogenase mit unterschiedlichen Mengen an Inhibitor

In Abbildung 8 sind die Aktivitätskurven der Lactat-Dehydrogenase mit unterschiedlichen NADH- und Inhibitor-Konzentrationen nach der Darstellungsweise von Lineweaver-Burk aufgezeichnet. Daraus ist zu entnehmen, daß mit steigender Inhibitorkonzentration einerseits die größtmögliche Reaktionsgeschwindigkeit (V) abnimmt.

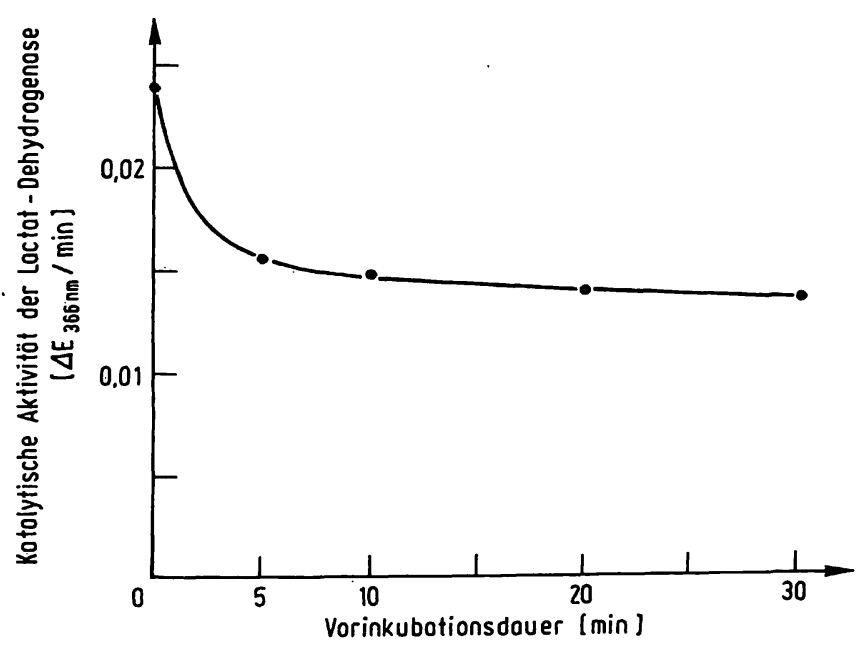

Abb. 7. Einfluß der Vorinkubationsdauer der Lactat-Dehydrogenase mit dem Oxidoreductase-Inhibitor auf die Hemmung der Anfangs-Aktivität

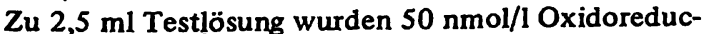
tase-Inhibitor und $0,1 \mathrm{ml}$ Enzymlösung zugemischt und bei Raumtemperatur vorinkubiert. Nach bestimmten Vorinkubationszeiten wurde die Enzymreaktion durch Zugabe des NADH gestartet und die Anfangs-Aktivität der Lactat-Dehydrogenase bei einer Inkubationstemperatur von $25^{\circ} \mathrm{C}$ bestimmt, indem die Extinktionsdifferenz während der ersten Minute bei der Wellenlänge $366 \mathrm{~nm}$ gemessen wurde. Ohne Inhibitor betrug $\Delta \mathrm{E}_{366 \mathrm{~nm} / \mathrm{min}}=0,045$. 


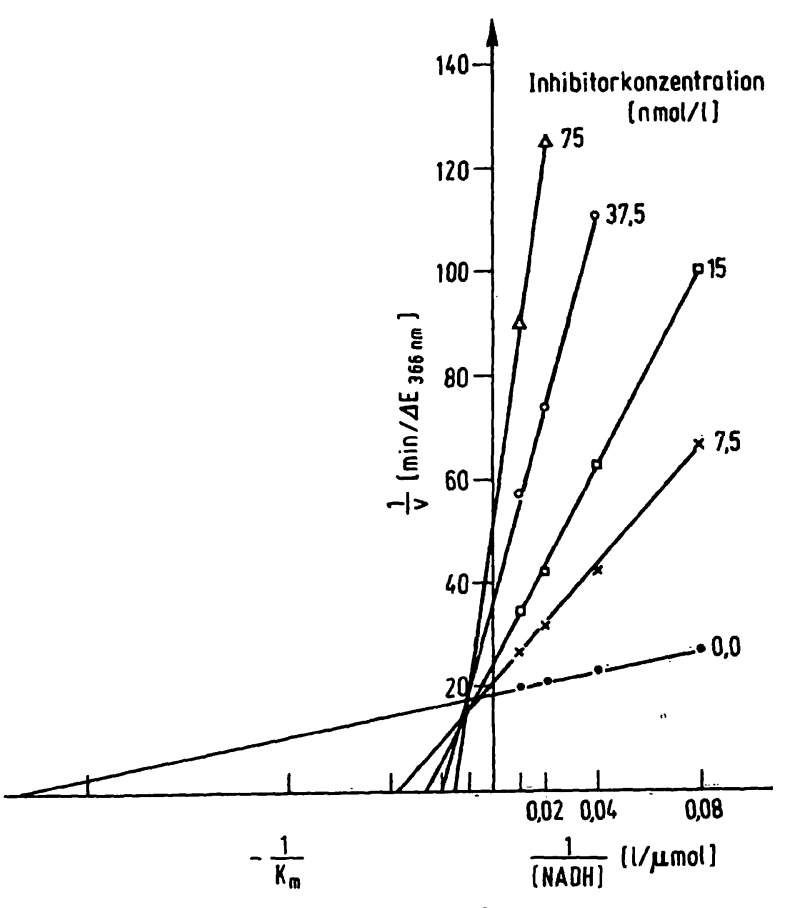

Abb. 8. Abhängigkeit der Lactat-Dehydrogenase-Aktivität von der NADH- und der Inhibitor-Konzentration: Darstellungsweise nach Lineweaver-Burk

Die Lactat-Dehydrogenase wurde während 10 Minuten bei $25^{\circ} \mathrm{C}$ ohne Inhibitor $(\cdot-)$, mit $7,5 \mathrm{nmol} / 1$ Inhibitor $(\mathrm{x}-\mathrm{x})$, mit $15 \mathrm{nmol} / 1$ Inhibitor ( $-\infty)$, mit $37,5 \mathrm{nmol} / 1$ Inhibitor $(0-0)$ und mit $75 \mathrm{nmol} / \mathrm{l}$ Inhibitor $(\Delta-\Delta)$ vorinkubiert. Nach Zugabe der angegebenen Menge NADH wurde die Anfangsaktivität

$\left(\Delta \mathrm{E}_{366 \mathrm{~nm} / \mathrm{min}}\right)$ bestimmt.

Bei dieser Darstellungsweise wird $1 / v$ ( $v=$ Reaktionsgeschwindigkeit als $\Delta \mathrm{E}_{366 \mathrm{~nm} / \mathrm{min}}$ ) in Funktion $\mathrm{zu} 1 /[\mathrm{S}]$ ([S] = NADH-Konzentration) gesetzt.

Die Werte der Abbildung 8 können auch nach der Methode von Dixon (12) graphisch dargestellt werden, indem die Reaktionsgeschwindigkeit der Lactat-Dehydrogenase bei einer bestimmten NADH-Konzentration reziprok gegen die eingesetzte Inhibitormenge aufgetragen wird (Abb. 9). Am Schnittpunkt der drei extrapolierten Reaktionslinien kann direkt die Inhibitorkonstante abgelesen werden: $K_{i}=3,5 \mathrm{nmol} / 1$.

Dialyseversuch des Oxidoreductase-Inhibitors bei Anwesenheit von Lactat-Dehydrogenase

Die Inhibitorkonstante von 3,5 nmol/1 bedeutet, daß der Oxidoreductase-Inhibitor eine äußerst starke Affinität zur Lactat-Dehydrogenase besitzt und eine entsprechend feste Bindung mit dem Enzym eingeht. Um die Frage abzuklären, ob unter diesen Umständen der OxidoreductaseInhibitor bei Anwesenheit von Lactat-Dehydrogenase noch dialysierbar ist oder ob er wegen dieser festen Bindung mit dem Enzym zurückgehalten wird, wurden folgende Versuche durchgeführt:

1. Die in der Tabelle 2 aufgefuhrten Komponenten wur-

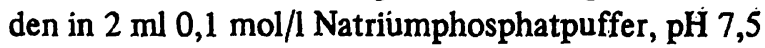
gelöst und in einen Dialysierschlauch eingefüllt. Die Proben wurden anschließend bei Raumtemperatur

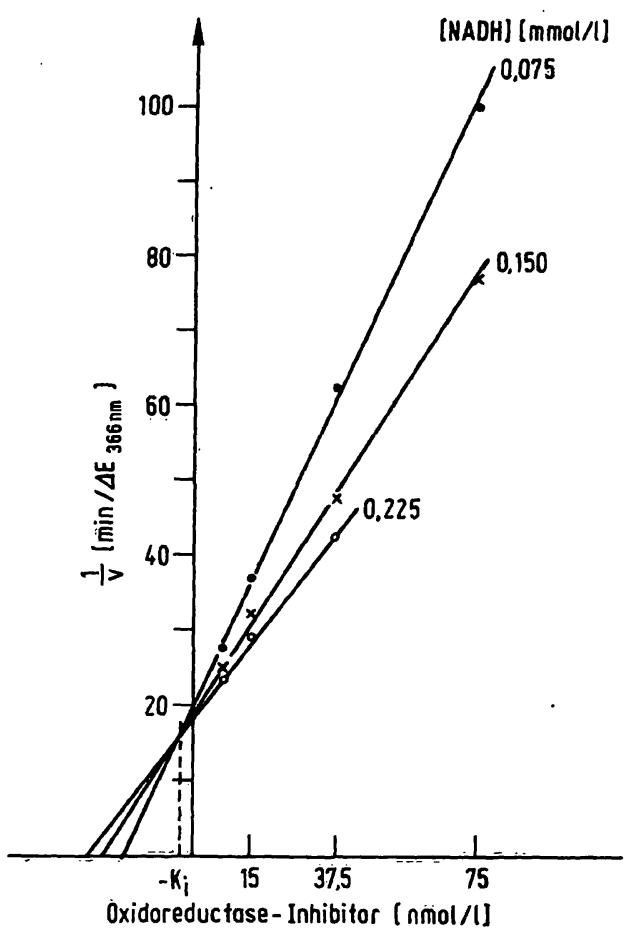

Abb. 9. Darstellung der Resultate der Abb. 8 nach der Methode von Dixon

Die Resultate der $\mathrm{Abb} .8$ wurden so aufgetragen, daß $1 / v\left(v=\right.$ Raktionsgeschwindigkeit $\left.\Delta \mathrm{E}_{366} \mathrm{~nm} / \mathrm{min}\right)$ in Funktion zur betreffenden Inhibitor-Konzenträtion gesetzt wurde. Der Schnittpunkt, der durch die drei extrapolierten Reaktionslinien gebildet wird, ergibt die Inhibitor-Konstante $\left(-\mathrm{K}_{\mathrm{i}}\right)$, deren Wert auf dër linken Basislinie direkt abgelesen werden kann.

Tab. 2. Dialyseversuch des Oxidoreductase-Inhibitors bei Anwesenheit von Lactat-Dehydrogenase

Die 8 aufgeführten Komponenten wurden in je $2 \mathrm{ml}$ $0,1 \mathrm{~mol} / 1$ Natriumphosphatpuffer, pH 7,5 gelöst und während 24 Stunden bei $25^{\circ} \mathrm{C}$ gegen $50 \mathrm{ml}$ derselben Pufferlösung im Text.
Im Dialyseschlauch

1. Oxidoreductase-Inhibitor

2. NADH

3. Oxidoreductase-Inhibitor + NADH

4. Lactat-Dehydrogenase + Oxidoreductase-Inhibitor

5. Lactat-Dehydrogenase + NADH

6. Lactat-Dehydrogenase + NADH + Inhibitor

7. Rinderserum-Albumin + Inhibitor

8. Human- $\gamma$-Globulin + Inhibitor

\section{Resultat}

Der Inhibitor ist dialysierbar Das NADH ist dialysierbar Beide Komponenten dialysieren

Der Inhibitor dialysiert nicht, die Lactat-Dehydrogenase im Dialyseschlauch bleibt gehemmt

Das NADH dialysiert

Das NADH dialysiert, während der Inhibitor im Dialyseschlauch bleibt und die LactatDehydrogenase hemmt Der Inhibitor dialysiert

Der Inhibitor dialysiert während 24 Stunden gegen $50 \mathrm{ml}$ 0,1 mol/1 Natriumphosphatpuffer, pH 7,5 dialysiert. Nach Abschluß der Dialyse wurde der Inhalt des Dialyseschlauchs sowie die Dialysierlösung auf den Gehalt der eingesetzten Kompo- 
nenten geprüft. Die Resultate dieses Versuchs sind in der Tabelle 2 zusammengestellt. Daraus ist ersichtlich, daß der Oxidoreductase-Inhibitor spezifisch von der Lactat-Dehydrogenase fest gebunden wird, so daß er nicht aus dem Dialyseschlauch in die Dialysierlösung hinausdiffundieren kann. Anderseits ist das NADH auch bei Anwesenheit von Lactat-Dehydrogenase leicht dialysierbar.

2. In einem zweiten Versuch wurde der OxidoreductaseInhibitor zur Dialysierlösung zugegeben (in $50 \mathrm{ml}$ $0,1 \mathrm{~mol} / 1$ Natriumphosphatpuffer, $\mathrm{pH} \mathrm{7,5)}$ und der Dialyseschlauch nur mit Lactat-Dehydrogenase (400 U in $2 \mathrm{ml}$ ) gefüllt. Die Dialyse wurde bei Raumtemperatur und unter leichtem Rühren durchgeführt. Nach bestimmten Zeitintervallen wurde in der Dialysierlösung der Gehalt an Ocidoreductase-Inhibitor gemessen und dabei eine fortlaufende Abnahme der Inhibitorkonzentration festgestellt. Nach der 48-stündigen Dialyse enthielt die Dialysierlösung noch $20 \%$ des eingesetzten Oxidoreductase-Inhibitors. Anderseits wies die Lactat-Dehydrogenase im Dialyseschlauch eine deutliche Aktivitätshemmung auf, so daß angenommen werden kann, daß der Inhibitor aus der Dialysierlösung in den Dialyseschlauch hineindiffundierte und dort von der Lactat-Dehydrogenase gebunden wurde.

\section{Diskussion}

Das Absorptionsspektrum des Oxidoreductase-Inhibitors zeigt im Unterschied zu jenem des NADH kein Extinktionsmaximum bei der Wellenlänge $340 \mathrm{~nm}$.

Bei der Aufnahme der Fluoreszenzspektren hat sich ergeben, daß im Gegensatz zum NADH die Fluoreszenz des Inhibitors durch Säure nicht verschwindet. Es werden weitere Arbeiten nötig sein, um abzuklären, wieweit dieses. unterschiedliche Verhalten zur Qualitätskontrolle des NADH herangezogen werden kann.

Der Oxidoreductase-Inhibitor kann durch eine DEAECellulose-Chromatographie vom NADH abgetrennt werden. Anderseits kann auch die Bildung des Inhibitors im NADH-Präparat dadurch verhindert werden, daß das Coenzym wasserfrei und ohne oxydierende Substanzen aufbewahrt wird. Versuche, den schon gebildeten Inhibitor im NADH-Präparat zu inaktivieren, sind wegen der hohen Stabilität des İnhibitors im alkalischen Milieu wie auch gegenüber organischen Löșungsmitteln erfolglos g̣eblie- ben. Es war auch nicht möglich, den Inhibitor durch $\mathrm{Zu}$ sätze von Kationen oder Aminosäuren zu binden und in seiner Hemmwirkung zu neutralisieren.

Der Oxidoreductase-Inhibitor hemmt vor allem und im gleichen Maß die Aktivität der fünf Isoenzyme der LactatDehydrogenase. Während die Malat-Dehydrogenase nur schwach gehemmt wird, übt der Inhibitos auf die Aktivität der übrigen untersuchten Oxidoreductasen keinen Einfluß aus.

Die Hitzedenaturierung der Lactat-Dehydrogenase bei einer Inkubationstemperatur von $65^{\circ} \mathrm{C}$ kann durch Zusätze von NADH oder auch von Oxidoreductase-Inhibitor wesentlich verlangsamt werden. Wegen der bedeutend höheren Affinität des Inhibitors zur LactatDehydrogenase braucht es von dieser Substanz eine entsprechend geringere Konzentration für eine gleiche Hitzestabilisierung, wie sie mit NADH erreicht wird.

Die Bindung des Oxidoreductase-Inhibitors an die LactatDehydrogenase wird bei Anwesenheit von NADH erschwert, so daß die volle Aktivitätshemmung der LactatDehydrogenase durch den eingesetzten Inhibitor erst verzögert wirksam wird. Wird aber die Lactat-Dehydrogenase zusammen mit dem Inhibitor vorinkubiert und die Reaktion anschließend durch Zugabe von NADH gestartet, so wird die Lactat-Dehydrogenase von Anfang an gehemmt. Offenbar bietet das NADH der Lactat-Dehydrogenase einen gewissen Schutz gegenüber dem Inhibitor, was vermuten läßt, daß die beiden Komponenten das Enzym an der gleichen Bindungsstelle angreifen.

Auf Grund der kinetischen Studien kann angenommen werden, daß es sich beim Oxidoreductase-Inhibitor um einen nicht-kompetitiven Hemmstoff handelt, dessen Inhibitorkonstante $3,5 \mathrm{nmol} / 1$ beträgt. Der Inhibitor besitzt somit eine äußerst hohe Affinität zur Lactat-Dehydrogenase, und er geht mit dem Enzym eine so stabile Bindung ein, daß er bei Anweșenheit von Lactat-Dehydrogenase nicht mehr dialysierbar ist.

Diese hohe Affinität des Oxidoreductase-Inhibitors zur Lactat-Dehydrogenase macht es verständlich, daß schon kleinste Spuren dieses Inhibitors in den NADH-Präparaten die Aktivitäts-Bestimmung der Lactat-Dehydrogenase wesentlich zu stören vermag. Anderseits wird es für die Qualitätskontrolle des NADH schwierig sein, den Inhibitorgehalt mit anderen Methoden als der Bestimmung der Aktivitätshemmung der Lactat-Dehydrogenase quạntitativ zu messen. 


\section{Literatur}

1. Fawcett, C., Ciotti, M. \& Kaplan, N. (1961), Biochim. Biophys. Acta 54, 210-212.

2. Fine, I., Fawcett, C., Everse, J. \& Kaplan, N. (1962), J. Amer. Chem. Soc. (Abstr.) 55C.

3. Dalziel, K. (1963), J. Biol. Chem. 238, 1538-1543.

4. Strandjord, P. \& Clayson, K. (1966), J. Lab. Clin. Med. 67, 144-153.

5. Babson, A. \& Arndt, E. (1970), Clin. Chem. 16, 254-255.

6. Berry, A., Lott, J. \& Grannis, G. (1973), Clin. Chem. 19, 1255-1258.
7. Gerhardt, W., Kofoed, B., Westlund, L. \& Pavlu, B. (1974), Scand. J. Clin. Lab. Invest. 33, 3-51.

8. Holman, M., Willis, J. \& Siegèl, J. (1966), Clin. Chem. 12, 543.

9. Klotzsch, S. \& Klotzsch, H. (1969), Clin. Chem. 15, $1056-1061$.

10. Gallati, H. (1976), diese Z. 14, 3-8.

11. Deutsche Gesellschaft für Klinische Chemie, (1972), diese $Z$. 10, 182-192.

12. Dixon, M. (1953), Biochem. J. 55, 170-171.

Dr. H. Gallati,

Diagnostische Forschungsabteilung, F. Hoffmann-La Roche \& Co, AG, Grenzacherstr. 124, CH-4002 Basel, Schweiz 